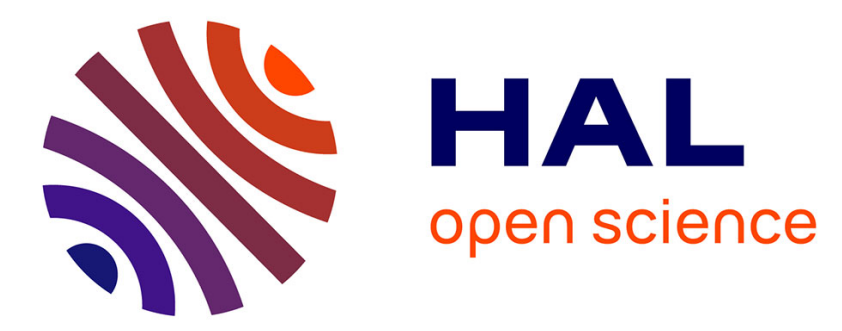

\title{
Quelques propriétés physico-chimiques des verres CdGe1-xSb xAs2
}

\author{
Nguyen van Dong, Tran Huu Danh, B. Auguin, A. Defresne
}

\section{To cite this version:}

Nguyen van Dong, Tran Huu Danh, B. Auguin, A. Defresne. Quelques propriétés physicochimiques des verres CdGe1-xSb xAs2. Revue de Physique Appliquée, 1977, 12 (5), pp.673-676. 10.1051/rphysap:01977001205067300 . jpa-00244223

\section{HAL Id: jpa-00244223 https://hal.science/jpa-00244223}

Submitted on 1 Jan 1977

HAL is a multi-disciplinary open access archive for the deposit and dissemination of scientific research documents, whether they are published or not. The documents may come from teaching and research institutions in France or abroad, or from public or private research centers.
L'archive ouverte pluridisciplinaire HAL, est destinée au dépôt et à la diffusion de documents scientifiques de niveau recherche, publiés ou non, émanant des établissements d'enseignement et de recherche français ou étrangers, des laboratoires publics ou privés. 


\title{
QUELQUES PROPRIÉTÉS PHYSICO-CHIMIQUES DES VERRES CdGe ${ }_{1-x} \mathrm{Sb}_{x} \mathrm{As}_{2}$
}

\author{
NGUYEN VAN DONG, TRAN HUU DANH, B. AUGUIN et A. DEFRESNE
}

Laboratoire de Physique des Matériaux, Service de Chimie-Physique, Centre d'Etudes Nucléaires de Saclay, 91190 Gif-sur-Yvette, France

\begin{abstract}
Résumé. - Des échantillons de verres du système $\mathrm{CdGe}_{1-x} \mathrm{Sb}_{x} \mathrm{As}_{2}$ avec $0 \leqslant x \leqslant 0,5$ ont été caractérisés par l'analyse thermique différentielle (A. T. D.) et les mesures de microdureté. La masse spécifique et la conductivité électrique ont été également mesurées pour différentes compositions. Les thermogrammes A. T. D. révèlent une diminution progressive des températures de transition vitreuse et de cristallisation. La microdureté décroît linéairement pour $0 \leqslant x \leqslant 0,3$ et tend à se stabiliser pour des compositions $x \geqslant 0,3$. La chaleur spécifique mesurée pour deux compositions $x=0$ et $x=0,5$ montre un accroissement brusque au voisinage de la température de transition vitreuse ; l'accroissement est moins élevé pour le composé contenant $\mathrm{Sb}$. Le processus de cristallisation dans les différents composés obéit approximativement à une cinétique du premier ordre. Le remplacement d'une concentration atomique de Ge par une même quantité de Sb conduit à une forte décroissance de la vitesse de cristallisation. L'enthalpie de cristallisation diminue légèrement quand la teneur en $\mathrm{Sb}$ augmente. Les observations au microscope optique et les mesures de microdureté sur les échantillons cristallisés révèlent l'apparition de plusieurs phases dans des composés contenant $\mathrm{Sb}$. La composition chimique de ces phases a été déterminée à l'aide de la microscopie électronique à balayage.
\end{abstract}

\begin{abstract}
Glassy compounds of the system $\mathrm{CdGe}_{1-x} \mathrm{Sb}_{x} \mathrm{As}_{2}$ with $x$ varying from 0 to 0.5 have been investigated by means of differential thermal analysis and microhardness measurements. The density and the electrical conductivity have been measured for different compounds. The thermograms show a progressive decrease of the glass transition and crystallization temperatures as the $\mathrm{Sb}$ content increases. The microhardness decreases linearly in the composition range $0 \leqslant x \leqslant 0.3$ and tends to be stabilized for higher composition. It is found that the heat capacity measured for two compounds $(x=0, x=0.5)$ increases abruptly near the glass transition; the increase is of less magnitude for compound containing $\mathrm{Sb}$. The crystallization process in different compounds is found to obey approximatively first-order kinetics. The partial substitution of $\mathrm{Sb}$ for $\mathrm{Ge}$ leads to a strong decrease of the crystallization rate. The enthalpy of crystallization is found to decrease slightly with the increase of $\mathrm{Sb}$ content. Optical microscope observations and microhardness measurements on crystallized compounds show the appearance of several phases for samples containing Sb. The chemical composition of these phases has been determined by means of scanning electron microscopy.
\end{abstract}

1. Introduction. - Les semiconducteurs amorphes tétraédriques de la famille $\mathrm{CdX}_{x} \mathrm{As}_{2}$ peuvent être obtenus par trempe à partir de l'état liquide. Les éléments $\mathrm{X}$ qui jouent en quelque sorte le rôle de vitrifiant pour le composé de base $\mathrm{CdAs}_{2}$ sont $\mathrm{Ge}$, $\mathrm{Si}, \mathrm{Tl}, \mathrm{In}, \mathrm{Ga}, \mathrm{Sb}, \mathrm{Al}, \mathrm{Mg}$ avec $x$ variant entre 0,05 et 1 mole environ. Ces composés sont susceptibles de présenter un intérêt technologique. En particulier, c'est le cas du $\mathrm{CdGeAs}_{2}$ amorphe dont les effets de commutation électronique ont été étudiés par Baryshev et al. [1].

Comme Ge et $\mathrm{Sb}$ peuvent vitrifier $\mathrm{CdAs}_{2}$, nous nous sommes proposés, en partant du $\mathrm{CdGeAs}_{2}$ amorphe, d'étudier l'effet d'une substitution progressive de $\mathrm{Sb}$ au $\mathrm{Ge}$ sur les caractéristiques thermiques [2] et les propriétés électroniques [3].

Le présent travail fait état d'une analyse plus détaillée des résultats obtenus sur les caractéristiques ther- miques que nous avons publiés ailleurs [2]. En plus, nous y exposerons l'étude expérimentale de la chaleur spécifique mesurée en calorimétrie différentielle et les résultats obtenus en microscopie électronique à balayage sur les composés recristallisés.

2. Méthodes et conditions expérimentales. - Les verres du système $\mathrm{CdGe}_{1-x} \mathrm{Sb}_{x} \mathrm{As}_{2}$ avec des compositions variant entre $x=0$ et $x=0,5$ ont été préparés à partir des éléments de haute pureté. Les éléments constituants étaient pesés et enfermés dans des ampoules de quartz scellées sous vide. Placées dans un four, les ampoules étaient chauffées lentement jusqu'à $850^{\circ} \mathrm{C}$, environ $200-250^{\circ} \mathrm{C}$ au-dessus de la température de fusion des composés. Un moteur électrique faisait tourner les ampoules pendant $15 \mathrm{~h}$ environ pour homogénéiser les liquides. Les composés fondus étaient ensuite trempés dans un bain d'eau froide. 
2.1 ANALYSE THERMIQUE ET CALORIMÉTRIE DIFFÉRENTIELLES. - Pour chaque composé, une quantité de $60 \mathrm{mg}$ était contenue dans une petite ampoule de quartz vidée et scellée. L'analyse thermique différentielle était effectuée à l'aide d'un analyseur thermique Setaram avec une vitesse de chauffe de 2 à $30^{\circ} \mathrm{C} \mathrm{min}^{-1}$. La chaleur spécifique $C_{\mathrm{p}}$ et l'enthalpie de cristallisation $\Delta H_{\mathrm{c}}$ étaient mesurées avec un calorimètre différentiel Mettler TA 2000, la cellule étant calibrée avec l'alumine standard. L'enthalpie de cristallisation était étalonnée par rapport à la chaleur de fusion de l'indium. La précision des mesures de $C_{\mathrm{p}}$ et de $\Delta H_{\mathrm{c}}$ est estimée à $5 \%$ environ.

2.2 Microdureté et MASSE SPÉCIFIQUe. - Les mesures de microdureté Vickers ont été effectuées sur des échantillons dont la surface était polie mécaniquement à la poudre de diamant et soumise à une attaque métallographique. Une charge de $100 \mathrm{~g}$ était utilisée pour faire l'empreinte. La masse spécifique était mesurée à l'aide d'un picnomètre à l'eau avec une précision d'environ $1 \%$.

3. Résultats expérimentaux et discussion. - L'état amorphe de nos échantillons dans l'intervalle de composition étudié $(0 \leqslant x \leqslant 0,5)$ était confirmé par la diffraction des rayons $X$. Le tableau 1 représente les trois températures de transformation $T_{\mathrm{g}}, T_{\mathrm{c}}, T_{\mathrm{f}}$ relatives à la transition vitreuse, à la cristallisation et à la fusion obtenues à l'aide de l'analyse thermique différentielle avec une vitesse de chauffe $8^{\circ} \mathrm{C} \min ^{-1}$.

\section{TABLEAU I}

Températures de transformation des composés $\mathrm{CdGe}_{1-x} \mathrm{Sb}_{x} \mathrm{As}_{2}$

$\begin{array}{cccc}\begin{array}{c}\text { Compo- } \\ \text { sition } x\end{array} & \begin{array}{c}\text { Température } \\ \text { de transition } \\ \text { vitreuse } \\ \left({ }^{\circ} \mathrm{C}\right)\end{array} & \begin{array}{c}\text { Température } \\ \text { de } \\ \text { cristallisation } \\ \left({ }^{\circ} \mathrm{C}\right)\end{array} & \begin{array}{c}\text { Température } \\ \text { de fusion } \\ \left({ }^{\circ} \mathrm{C}\right)\end{array} \\ - & - & - & - \\ 0 & 386 & 440 & 666 \\ 0,1 & 370 & 430 & 650 \\ 0,3 & 345 & 420 & 615 \\ 0,5 & 315 & 395 & 527 \\ & & & 557\end{array}$

Pour la composition $x=0,5$, nous avons signalé deux transitions vitreuses dont la première aurait lieu à $150^{\circ} \mathrm{C}$ [2]. Cependant, les mesures en calorimétrie différentielle n'ont pas confirmé l'existence de cette première transition vitreuse.

Les variations de la température de transition vitreuse $T_{\mathrm{g}}$ avec la composition sont représentées sur la figure 1. Nous constatons une diminution linéaire de $T_{\mathrm{g}}$ quand la teneur en $\mathrm{Sb}$ augmente. La courbe $1 a$ représente les résultats obtenus par Gorjunova et al. [4] sur les composés $\mathrm{CdGe}_{1-x} \mathrm{As}_{2}$. Le glissement de $T_{\mathrm{g}}$ vers les basses températures correspondant à la diminution de la teneur en Ge suggère l'existence des liaisons Ge-As. La courbe $1 b$ relative à nos échantillons montre clairement que l'addition de $\mathrm{Sb}$ a pour effet

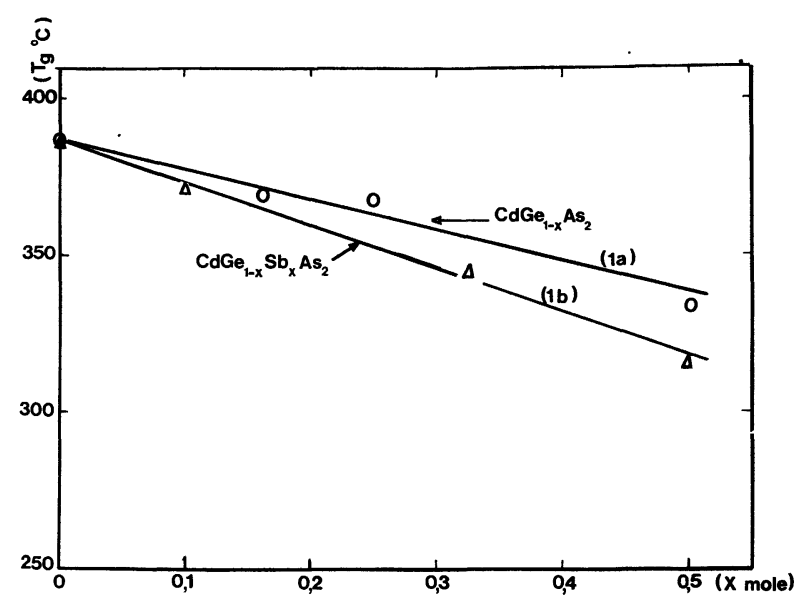

Fig. 1. - Variations de la température de transition vitreuse en fonction de la composition. La courbe $1 a$ est relative au $\mathrm{CdGe}_{1-x} \mathrm{As}_{2}$ d'après les résultats de Gorjunova et al. [4].

d'abaisser $T_{\mathfrak{g}}$. Ce fait laisse à supposer la présence des liaisons Sb-As à côté des liaisons Ge-As dans ces composés. De même, la diminution progressive de la microdureté en fonction de la teneur en $\mathrm{Sb}$ peut être expliquée sur la base de cette hypothèse. Cependant, pour des compositions $x \geqslant 0,3$ la microdureté a tendance à se stabiliser.

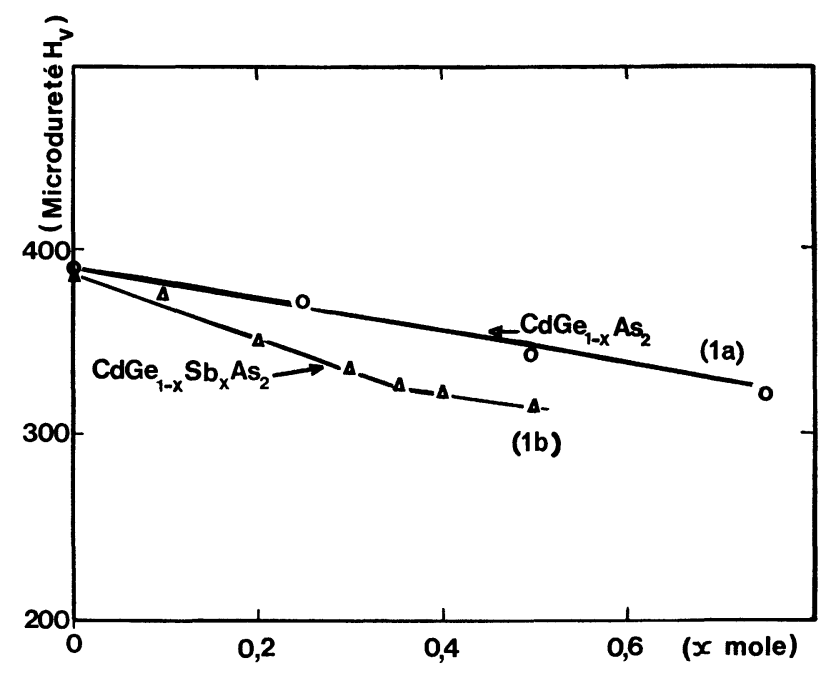

FIG. 2. - Variations de la microdureté en fonction de la composition. La courbe $1 a$ est relative au $\mathrm{CdGe}_{1-x} \mathrm{As}_{2}$, d'après Gorjunova et al. [4].

Les valeurs de la masse spécifique et de la conductivité électrique pour différents composés sont donnés dans le tableau II. Le léger accroissement de la masse spécifique avec l'augmentation de la teneur en $\mathrm{Sb}$ s'explique par la différence de masse atomique entre le $\mathrm{Sb}$ et le Ge. Les variations de la conductivité électrique à la température ambiante en fonction de la teneur en $\mathrm{Sb}$ correspondent aux variations du gap d'énergie que nous avons déterminé [3]. 
TABLEAU II

$\begin{array}{cccc}\begin{array}{c}\text { Composition } \\ x\end{array} & \begin{array}{c}\text { Microdureté } \\ H_{\mathrm{v}}\end{array} & \begin{array}{c}\text { Masse } \\ \text { spécifique } \\ \mathrm{gcm}^{-3}\end{array} & \begin{array}{c}\text { Conductivité } \\ \text { électrique } \\ \Omega^{-1} \mathrm{~cm}^{-1}\end{array} \\ - & - & - & - \\ 0 & 385 & 5,74 & 6,6 \times 10^{-7} \\ 0,1 & 380 & 5,74 & 1,8 \times 10^{-6} \\ 0,3 & 330 & 5,77 & 5,2 \times 10^{-6} \\ 0,5 & 314 & 5,82 & 2 \times 10^{-5}\end{array}$

Pour étudier la chaleur spécifique dans un domaine de température couvrant la température de transition vitreuse, nous avons effectué des mesures en calorimétrie différentielle sur deux compositions extrêmes $(x=0, x=0,5)$. La figure 3 représente les thermo-

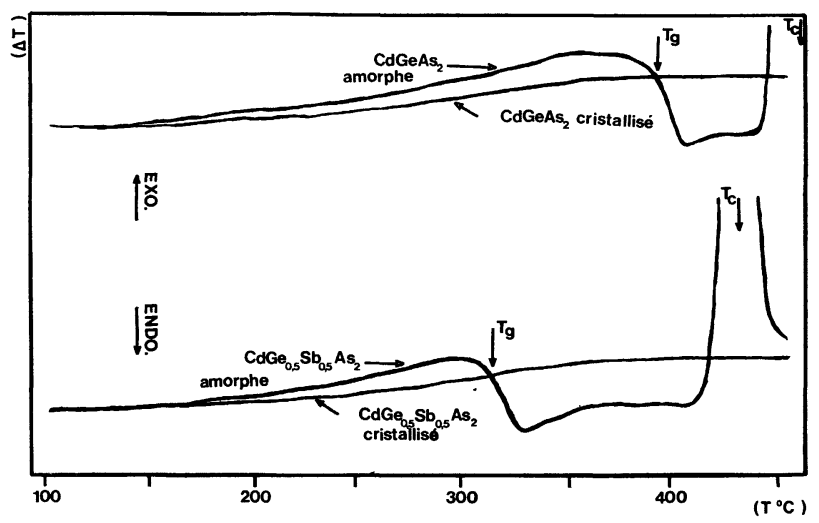

Fig. 3. - Thermogramme obtenu en calorimétrie différentielle pour deux composés $\mathrm{CdGeAs}_{2}$ et $\mathrm{CdGe}_{0,5} \mathrm{Sb}_{0,5} \mathrm{As}_{2}$. Poids pour chaque composé $25 \mathrm{mg}$. Vitesse de chauffe $10^{\circ} \mathrm{C} \mathrm{m^{-1 }}$.

grammes obtenus avec une vitesse de chauffe $10^{\circ} \mathrm{C}$ $\min ^{-1}$. On constate pour deux composés un phénomène exothermique étalé sur un intervalle de température relativement grand, puis après $T_{\mathrm{g}}$ un pic exothermique quasi explosif accompagnant la cristallisation. Le premier phénomène exothermique résulte probablement du développement de germination. Pour le $\mathrm{CdGeAs}_{2}$, les observations au microscope optique [5] ont montré en effet que le processus de nucléation se produit dans l'intervalle de température correspondant approximativement au phénomène exothermique observé ici. Pour le $\mathrm{CdGe}_{0,5} \mathrm{Sb}_{0,5} \mathrm{As}_{2}$, dont la température de transition est moins élevée, le phénomène est de moindre importance.

La figure 4 représente la chaleur spécifique en fonction de la température pour les composés en question. On constate que la chaleur spécifique dans l'état amorphe et l'état cristallisé sont les mêmes au-dessous de $100{ }^{\circ} \mathrm{C}$, ce qui montre que $C_{\mathrm{p}}$ est due uniquement aux modes de vibration du réseau. La décroissance de $C_{\mathrm{p}}$ dans l'état amorphe à partir de $100^{\circ} \mathrm{C}$ est attribuée, comme nous l'avons dit précédemment, au processus de germination. L'accroissement brutal de $C_{\mathrm{p}}$ à la température de transition vitreuse est dû à l'introduction des modes de vibration supplémentaires résultant de la rupture des liaisons faibles. L'accroissement est moins élevé pour le composé contenant $\mathrm{Sb}$.

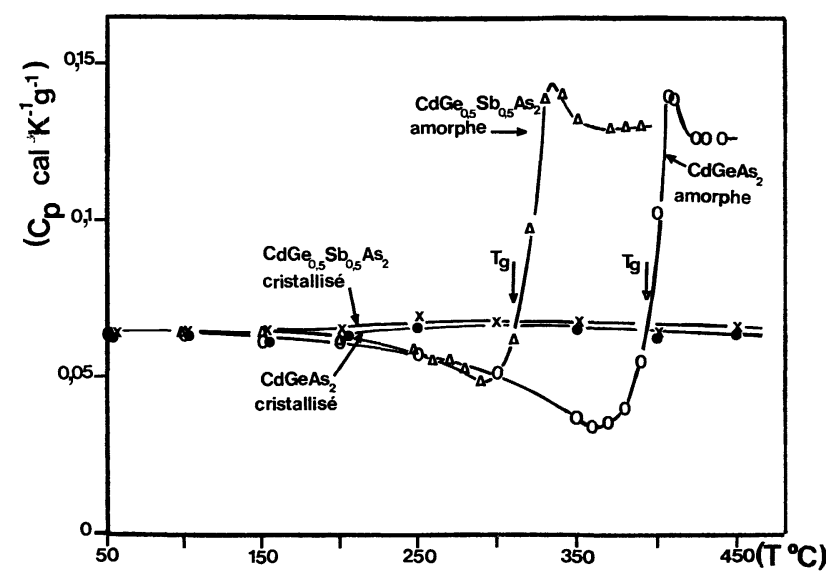

Fig. 4. - Variations de la chaleur spécifique en fonction de la température pour deux composés $\mathrm{CdGeAs}_{2}$ et $\mathrm{CdGe}_{0,5} \mathrm{Sb}_{0,5} \mathrm{As}_{2}$.

Nous avons enfin étudié le processus de cristallisation dans les différents composés en utilisant la méthode de Kissinger [6]. D'autres chercheurs ont récemment appliqué cette méthode à l'étude de la cristallisation dans les verres $\mathrm{CdGeAs}_{2}$ [7] et $\mathrm{As}_{2} \mathrm{Se}_{3}$ [8]. La méthode consiste à mesurer par analyse thermique différentielle les variations de la température de cristallisation avec la vitesse de chauffe.

Soit $f$ la partie cristallisée du matériau. Le taux de cristallisation peut s'exprimer par l'équation :

$$
\frac{\mathrm{d} f}{\mathrm{~d} t}=c(1-f)
$$

où $c$ est donné par :

$$
c=c_{\mathrm{o}} \exp \left(-E_{\mathrm{a}} / k T\right)
$$

$E_{\mathrm{a}}$ étant l'énergie d'activation de cristallisation et $C_{\mathrm{o}}$ un facteur de fréquence.

Quand le taux de cristallisation est maximum, on a :

$$
c_{\mathrm{o}} \exp \left(-E_{\mathrm{a}} / k T_{\mathrm{e}}\right)=\frac{E_{\mathrm{a}} r}{k T_{\mathrm{c}}^{2}}
$$

où $T_{\mathrm{c}}$ est la température correspondant au pic de cristallisation et $r$ la vitesse de chauffe. En traçant $\ln \left(r / T_{\mathrm{c}}{ }^{2}\right)$ en fonction de $1 / T$, on obtient une droite à partir de laquelle on déterminera $E_{\mathrm{a}}$ et $c_{\mathrm{o}}$. La figure 5 représente les résultats obtenus pour différents composés. Pour chaque composition, la plupart des points se trouvent sur une droite, ce qui montre que la cristallisation obéit à une cinétique du premier ordre. Ce résultat suggère qu'un processus de croissance par diffusion contrôle la cristallisation. Les valeurs de $E_{\mathbf{a}}$ et de $c_{\mathrm{o}}$ déterminées à partir des courbes expérimentales sont données dans le tableau III. Pour le $\mathrm{CdGeAs}_{2}$, la valeur de $E_{\mathrm{a}}$ est en bon accord avec celle obtenue par Risbud [7].

On constate que l'énergie d'activation de cristallisation $\mathrm{E}_{\mathrm{a}}$ diminue lorsque la teneur en $\mathrm{Sb}$ augmente. La diminution de $E_{a}$ peut s'expliquer de la façon suivante. Au-dessus de la température de transition, la 


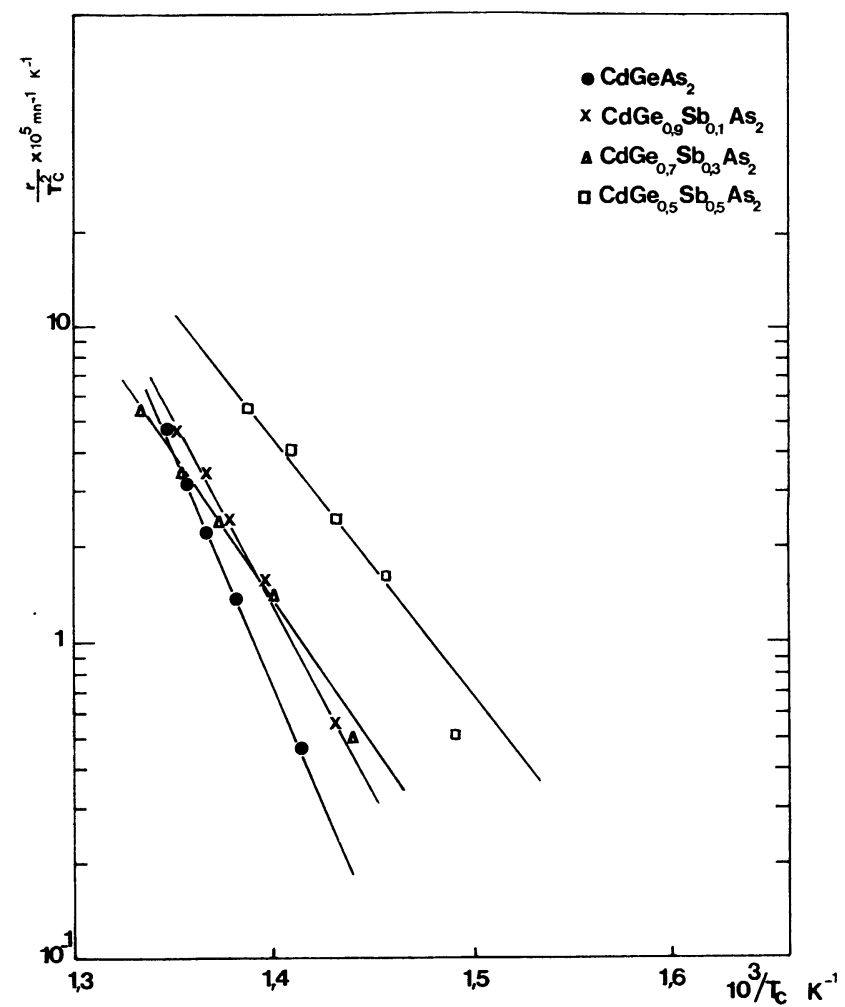

FIG. 5. - Courbes de cristallisation obtenues avec des vitesses de chauffe différentes.

\section{TABLEAU III}

$\begin{array}{ccc}\begin{array}{c}\text { Composition } \\ x\end{array} & \begin{array}{c}E_{\mathrm{a}} \\ \mathrm{eV}\end{array} & \begin{array}{c}c_{\mathrm{o}} \\ -\end{array} \\ - & -1,0 & 1,3 \times 10^{18} \\ 0 & 2,3 & 1,4 \times 10^{14} \\ 0,1 & 1,8 & 1,6 \times 10^{10} \\ 0,3 & 1,6 & 3,5 \times 10^{9} \\ 0,5 & & \end{array}$

séparation de phases aurait lieu probablement dans les composés contenant $\mathrm{Sb}$, donnant naissance à des amas de molécules de dimensions et de poids plus faibles et par conséquent de mobilité plus petite par comparaison avec ceux de molécules $\mathrm{CdGeAs}_{2}$. Les observations au microscope optique et les mesures de microdureté après cristallisation ont montré la présence de plusieurs phases dans les échantillons contenant $\mathrm{Sb}$.
L'analyse au microscope électronique à balayage a permis d'identifier ces phases. Dans les composés riches en $\mathrm{Sb}(x \geqslant 0,3)$, nous avons vu apparaître les phases ( $\mathrm{Sb}$, As) et $\mathrm{Cd}_{2} \mathrm{GeAs}_{4}$ à côté de la phase $\mathrm{CdGeAs}_{2}$. Mais l'addition de $\mathrm{Sb}$ a encore un effet plus considérable sur le facteur de fréquence $C_{\mathrm{o}}$ dont la valeur diminue de plusieurs ordres de grandeur. La réduction de $C_{\mathrm{o}}$ suggère que le nombre de nucléides dans les échantillons contenant $\mathrm{Sb}$ est plus faible. Ce résultat est en accord avec le fait que le phénomène exothermique se produisant avant la cristallisation dans le composé $\mathrm{CdGe}_{0,5} \mathrm{Sb}_{0,5}$ est de moindre importance que celui observé dans $\mathrm{CdGeAs}_{2}$. L'enthalpie de cristallisation $\Delta h_{\mathrm{c}}$ mesurée en calorimétrie différentielle sur deux compositions extrêmes $x=0$ et $x=0,5$ a été trouvée respectivement égale à $0,36 \mathrm{eV} / \mathrm{mol}$ et $0,32 \mathrm{eV} / \mathrm{mol}$ soit une valeur légèrement plus faible pour le composé contenant $\mathrm{Sb}$. La valeur moyenne est en assez bon accord avec celle que nous avons obtenue auparavant pour quatre compositions à l'aide d'un autre calorimètre [2]. Le fait que l'enthalpie de cristallisation ne dépend guère de la composition peut suggérer que la structure des divers composés amorphes étudiés serait construite autour du tétraèdre $\mathrm{CdAs}_{4}$, conformément à l'hypothèse de Cervinka et al sur la structure du $\mathrm{CdGeAs}_{2}$ [9].

4. Conclusion. - La substitution partielle des atomes de $\mathrm{Sb}$ aux atomes de $\mathrm{Ge}$ dans les verres du système $\mathrm{CdGe}_{1-x} \mathrm{Sb}_{x} \mathrm{As}_{2}$ conduit à un changement progressif de certaines propriétés physicochimiques étudiées dans ce travail. Ce comportement laisse à penser que l'unité de base, probablement le tétraèdre $\mathrm{CdAs}_{4}$, serait la même pour les divers composés. Les valeurs relativement élevées de la température de transition vitreuse, de microdureté et de l'énergie d'activation de cristallisation suggèrent que ces verres sont relativement stables et qu'ils peuvent, par conséquent, présenter un intérêt pour les applications en commutation électronique.

Remerciements. - Les mesures de microdureté et les observations au microscope optique ont été effectuées par MM. P. Maire et H. Hasson. Les échantillons ont été examinés au microscope électronique à balayage par M. P. Maurice. Nous tenons à leur exprimer ici nos vifs remerciements.

\section{Bibliographie}

[1] Baryshev, V. G., Boltivets, N. S., BorshchevskiI, A. S., Gorjunova, N. A. and Oreshrin, P. T., Sov. Phys. Semicond. 4 (1970) 398.

Chigogidze, Z. H., Kvirikasvili, T. Sh., BorshchevskiI, A. S., Ksaverieva, N. V. and Davidov, S. E., Soobshch. Akad. Nauk. Gruz. SSR, 76 (1974) 581.

[2] Nguyen Van Dong, Tran Huu Danh, Auguin, B. and DEFRESNE, A., 4th International Conference on The Phys. Non-Crystall. Solids (Clausthal-Zellerfeld) 1976.

[3] Nguyen Van Dong, Tran Huu Danh, Auguin, B. and Defresne, A., Phys. Stat. Solids 33 (1976) 295 ; 6th International Conference on Amorphous and Liquid Semiconductors, Leningrad, USSR (1975).

[4] Gorjunova, N. A., Radantsan, S. I., Kusmenco, G. S. and LJALIKova, R. J., 5th International Conference on Amorphous and Liquid Semiconductors, Garmisch, Partenkirchen (1973).

[5] Risbud, S., J. Amer. Ceram. Soc. 57 (1974) 272.

[6] Kissinger, H. E., J. Res. Nat. Bur. Stand. 57 (1956) 217.

[7] Risbud, S., J. Amer. Ceram. Soc. 56 (1973) 440.

[8] ThornburG, D. D., Johnson, R. I., J. Non-Crystall. Solids 17 (1975) 8.

[9] CervinkA, L., J. Non-Crystall. Solids 3 (1970) 294. 